\title{
ON MEAN OUTER RADII OF RANDOM POLYTOPES
}

\author{
D. ALONSO-GUTIÉRREZ, N. DAFNIS, M. Á. HERNÁNDEZ CIFRE, AND J. PROCHNO
}

\begin{abstract}
In this paper we introduce a new sequence of quantities for random polytopes. Let $K_{N}=\operatorname{conv}\left\{X_{1}, \ldots, X_{N}\right\}$ be a random polytope generated by independent random vectors uniformly distributed in an isotropic convex body $K$ of $\mathbb{R}^{n}$. We prove that the so-called $k$-th mean outer radius $\widetilde{R}_{k}\left(K_{N}\right)$ has order $\max \{\sqrt{k}, \sqrt{\log N}\} L_{K}$ with high probability if $n^{2} \leq N \leq e^{\sqrt{n}}$. We also show that this is also the right order of the expected value of $\widetilde{R}_{k}\left(K_{N}\right)$ in the full range $n \leq N \leq e^{\sqrt{n}}$.
\end{abstract}

\section{Introduction AND Notation}

The study of random polytopes began with Sylvester and the famous four-point problem nearly 150 years ago and it represents the starting point for an extensive study. In 1963, Rényi and Sulanke [31] continued it in their innovative work, studying expectations of various basic functionals of random polytopes. Important quantities are expectations, variances and distributions of those functionals. The tools combine a variety of mathematical disciplines such as convex geometry, geometric analysis or geometric probability (see also $[4,30]$ and the references therein).

Random polytopes appear in many important applications and other fields, e.g., in statistics (extreme points of random samples), convex geometry (approximation of convex sets), but also in computer science in the analysis of the average complexity of algorithms [28], optimization [6], and even in biology [32]. These are several reasons why in the last 30 years random polytopes have gained more and more interest.

Important and very recent developments in the study of random polytopes can be found in [20], where the authors prove that the isotropic constant of a Gaussian random polytope is bounded with high probability, and in $[1,2,3,9,10,11,27]$ where the authors study the relation between several parameters of a random polytope in an isotropic convex body and the isotropic constant of the body.

2000 Mathematics Subject Classification. Primary 52A22, Secondary 52A23, 05D40.

Key words and phrases. Mean Outer Radii, Random Polytope, Isotropic Constant.

Part of this work was done while the first, second and fourth authors were postdoctoral fellows at the Department of Mathematical and Statistical Sciences at the University of Alberta in Edmonton under the supervision of N. Tomczak-Jaegermann and A. Litvak. Second author is supported by the Action "Supporting Postdoctoral Researchers" of the Operational Program "Education and Lifelong Learning" (Action's Beneficiary: General Secretariat for Research and Technology), and is co-financed by the European Social Fund (ESF) and the Greek State. First and third authors are partially supported by MICINN project MTM2010-16679, MICINN-FEDER project MTM2009-10418 and "Programa de Ayudas a Grupos de Excelencia de la Región de Murcia", Fundación Séneca, 04540/GERM/06. Fourth author is supported by the Austrian Science Fund (FWF) within the FWF project P23987 "Projection operators in Analysis and geometry of classical Banach spaces". 
The main goal of this paper is to estimate a new sequence of quantities for random polytopes defined by $N$ random points in an isotropic convex body in $\mathbb{R}^{n}$, namely, a certain family of outer radii. In the last years, the so-called successive outer radii have been intensively studied, e.g. their geometric and analytical properties, size for special bodies, relation with other measures, and computational aspects (see e.g. $[5,7,14,15,16,17,18,29]$ and the references inside). These families of quantities are defined as the maximum/minimum of the (classical) circumradii of the projections of a convex body onto all $k$-dimensional subspaces. If we restrict to the family of polytopes, there is not too much information (see e.g. $[8,16]$ and, in particular, [7], where the precise values of -among others- the successive outer radii of the regular cube, cross-polytope and simplex can be found).

So, it is a natural generalization to consider a kind of mean outer radii. In the context of random polytopes, we will define them as the outer radius of the projection of the random polytope onto a subspace $F$ of dimension $k$, averaged over the Grassmannian manifold $G_{n, k}, 1 \leq k \leq n$, with respect to the Haar probability measure, and we will prove that, with high probability, this quantity has order $\max \{\sqrt{k}, \sqrt{\log N}\} L_{K}$, where $L_{K}$ is the isotropic constant of $K$.

We will work in $\mathbb{R}^{n}$ equipped with a Euclidean structure $\langle\cdot, \cdot\rangle$. We denote by $|\cdot|$ the corresponding Euclidean norm, as well as the volume (i.e., the Lebesgue measure) in $\mathbb{R}^{n}$. A convex body $K \subset \mathbb{R}^{n}$ is a compact convex set with non-empty interior, and it is called (centrally) symmetric if $-x \in K$, whenever $x \in K$. We write $B_{2}^{n}$ to denote the Euclidean unit ball, $S^{n-1}=\left\{x \in \mathbb{R}^{n}:|x|=1\right\}$ for the unit sphere in $\mathbb{R}^{n}$ and $\sigma$ for the uniform probability measure on $S^{n-1}$. Moreover, if $F \subset \mathbb{R}^{n}$ is a $k$-dimensional linear subspace, we denote by $S_{F}^{k-1}$ and $\sigma_{F}$ the corresponding unit sphere and probability measure in $F$, respectively.

We use the notation $a \simeq b$ to denote that there exist absolute constants $c_{1}>0$, $c_{2}>0$ such that $c_{1} a \leq b \leq c_{2} a$.

A convex body $K$ is isotropic if $|K|=1$, its centroid lies at the origin, i.e., $\int_{K} x d x=0$, and it satisfies the isotropic condition

$$
\int_{K}\langle x, \theta\rangle^{2} d x=L_{K}^{2}, \quad \text { for all } \theta \in S^{n-1},
$$

where $L_{K}$ is a constant depending only on $K$, the so-called isotropic constant of $K$. We refer to $[12,23]$ for further background information and results on isotropic convex bodies.

For a given convex body $K \subset \mathbb{R}^{n}$ and independent random vectors $X_{1}, \ldots, X_{N}$, uniformly distributed in $K$, we denote the corresponding random polytopes by

$$
K_{N}=\operatorname{conv}\left\{X_{1}, \ldots, X_{N}\right\} \quad \text { and } S_{N}=\operatorname{conv}\left\{ \pm X_{1}, \ldots, \pm X_{N}\right\} .
$$

Moreover, if $0 \in \operatorname{int} K$, the outer radius of $K$ is defined as the quantity

$$
R(K)=\min \left\{t>0: K \subseteq t B_{2}^{n}\right\}=\max _{x \in K}|x| .
$$

We observe that if $K$ is symmetric, then $R(K)$ coincides with the classical circumradius of $K$, namely, $\min \left\{R: \exists x \in \mathbb{R}^{n}\right.$ with $\left.K \subseteq x+R B_{2}^{n}\right\}$.

This magnitude leads to the definition of the main concept of this paper. For a convex body $K \subseteq \mathbb{R}^{n}$, the $k$-th mean outer radius of $K, 1 \leq k \leq n$, is defined as

$$
\widetilde{R}_{k}(K)=\int_{G_{n, k}} R\left(P_{F} K\right) d \nu_{n, k}(F),
$$


where $G_{n, k}$ denotes the set of $k$-dimensional linear subspaces of $\mathbb{R}^{n}, \nu_{n, k}$ is the unique Haar probability measure on $G_{n, k}$ invariant under orthogonal maps, and $P_{F} K$ is the orthogonal projection of $K$ onto $F$. We will write $d \nu_{F, k}$ if we work with the Grassmannian manifold $G_{F, k}$ restricted to a fixed subspace $F$. Clearly $\widetilde{R}_{n}(K)$ is the outer radius of $K$ and, moreover, if $K$ is symmetric, then $\widetilde{R}_{1}(K)=\omega(K)$ is the mean width of $K$ and $\widetilde{R}_{n}(K)$ its classical circumradius. We also observe that for the polytopes $K_{N}$ and $S_{N}$ and every $F \in G_{n, k}, 1 \leq k \leq n$, we have

$$
R\left(P_{F} K_{N}\right)=R\left(P_{F} S_{N}\right)=\max _{1 \leq j \leq N}\left|P_{F} X_{j}\right| \text {. }
$$

Hence, all results in this paper are valid for both $K_{N}$ and $S_{N}$, and so it suffices to deal only with one of them, say $K_{N}$.

The main result of this paper is an asymptotic formula which gives the right order for the mean outer radii of a random polytope lying in an isotropic convex body, with high probability:

Theorem 1.1. Let $K \subset \mathbb{R}^{n}$ be an isotropic convex body and $K_{N}$ be a random polytope generated by independent random vectors $X_{1}, \ldots, X_{N}$, uniformly distributed in $K$. If $n \leq N \leq e^{\sqrt{n}}$ then, for all $1 \leq k \leq n$ and $s>0$,

$$
c_{1}(s) \max \left\{\sqrt{k}, \sqrt{\log \frac{N}{n}}\right\} L_{K} \leq \widetilde{R}_{k}\left(K_{N}\right) \leq c_{2}(s) \max \{\sqrt{k}, \sqrt{\log N}\} L_{K}
$$

with probability greater than $1-N^{-s}$, where $c_{1}(s)$ and $c_{2}(s)$ are positive absolute constants depending only on $s$. In particular, if $n^{2} \leq N \leq e^{\sqrt{n}}$, then both the upper and the lower bounds have the same order, i.e.,

$$
\widetilde{R}_{k}\left(K_{N}\right) \simeq \max \{\sqrt{k}, \sqrt{\log N}\} L_{K}
$$

In order to prove Theorem 1.1, we modify some arguments appearing in [10, 11], and use in a crucial way two strong inequalities of Paouris (see [25, 26]). We observe (see Remark 4.1) that we cannot expect to extend the range for $N$ from above in estimate (2) with high probability. On the other hand, even though we can extend this range of $N$ from below, we cannot obtain this estimate with high probability when $N$ is proportional to $n$ with the techniques we use. However, this is feasible if the expectation is involved:

Theorem 1.2. Let $K \subset \mathbb{R}^{n}$ be an isotropic convex body and $K_{N}$ be a random polytope generated by independent random vectors $X_{1}, \ldots, X_{N}$, uniformly distributed in $K$. Then, for $n \leq N \leq e^{\sqrt{n}}$ and all $1 \leq k \leq n$,

$$
\mathbb{E} \widetilde{R}_{k}\left(K_{N}\right) \simeq \max \{\sqrt{k}, \sqrt{\log N}\} L_{K}
$$

We mention here that using a new method, in [3] the authors established a sharp lower estimate for the expectation of the mean width of a (symmetric) random polytope inside an isotropic convex body, for the range $N \simeq n$. We will use their result in the proof of Theorem 1.1 in order to show Theorem 1.2.

The paper is organized as follows. In Section 2 we state additional notation and known results that we will use throughout the paper. Section 3 is devoted to show several technical lemmas which will be needed in the proofs of the theorems. Finally, in Section 4 we prove Theorems 1.1 and 1.2. We conclude the paper showing in 
Section 5 the following result, in the flavor of Theorem 1.2, for Gaussian random polytopes:

Theorem 1.3. Let $X_{1}, \ldots, X_{N}$ be independent standard Gaussian random vectors in $\mathbb{R}^{n}, n \leq N$, and let $K_{N}=\operatorname{conv}\left\{X_{1}, \ldots, X_{N}\right\}$. Then, for all $1 \leq k \leq n$,

$$
\mathbb{E} \widetilde{R}_{k}\left(K_{N}\right) \simeq \max \{\sqrt{k}, \sqrt{\log N}\}
$$

\section{AdDitional notation And BACKGround Results}

The following definition can be found in [25] (see also [26]). Let $K$ be a convex body in $\mathbb{R}^{n}$ with volume one. For every $-n<p<+\infty, p \neq 0$, and every $F \in G_{n, k}$, the $p$-moment of the Euclidean norm of the projection of $K$ onto $F$ is defined as

$$
I_{p}(K, F)=\left(\int_{K}\left|P_{F} x\right|^{p} d x\right)^{1 / p} .
$$

If $F=\mathbb{R}^{n}$ we write

$$
I_{p}(K)=I_{p}\left(K, \mathbb{R}^{n}\right)=\left(\int_{K}|x|^{p} d x\right)^{1 / p} .
$$

In [25], Paouris proved a sharp reverse Hölder type inequality for the moments of the Euclidean norm of an isotropic convex body $K$ in $\mathbb{R}^{n}$ :

$$
I_{q}(K) \simeq I_{2}(K)=\sqrt{n} L_{K}, \quad \text { for all } 1 \leq q \leq \sqrt{n} .
$$

This inequality provides a sharp concentration result for the mass distribution in an isotropic convex body in $\mathbb{R}^{n}$, i.e., for any $t>0$,

$$
\mathbb{P}\left(|x| \geq c t \sqrt{n} L_{K}\right) \leq e^{-\sqrt{n} t} .
$$

We will need this result for the proof of the upper bound in Theorem 1.1.

In [26], the same author got the following small ball probability result for isotropic random vectors, i.e., centered random vectors $X$ verifying that $\mathbb{E}\langle X, \theta\rangle^{2}=1$ for any $\theta \in S^{n-1}$ :

Theorem 2.1 (Paouris, [26]). Let $X$ be an isotropic log-concave random vector in $\mathbb{R}^{n}$. Let $M$ be a non-zero $n \times n$ matrix, $y \in \mathbb{R}^{n}$ and $\varepsilon \in(0,1)$. Then

$$
\mathbb{P}\left(|M X-y| \leq c_{1} \varepsilon\|M\|_{\mathrm{HS}}\right) \leq \varepsilon^{c_{2} \frac{\|M\|_{\mathrm{HS}}}{\|M\|_{\mathrm{op}}}},
$$

where $c_{1}, c_{2}$ are positive absolute constants and $\|M\|_{\mathrm{HS}},\|M\|_{\mathrm{op}}$ denote the HilbertSmith and the operator norm of $M$, respectively.

In order to show the theorem, the author proved a reverse Hölder inequality for the negative moments of the Euclidean norm on an isotropic convex body $K \subset \mathbb{R}^{n}$ :

$$
I_{-q}(K) \simeq I_{2}(K)=\sqrt{n} L_{K}, \quad \text { for all } 1 \leq q \leq \sqrt{n}
$$

For the proof of the lower bound in Theorem 1.1 we need some more background. For a convex body $K \subset \mathbb{R}^{n}, 0 \in \operatorname{int} K$, and every $-\infty<p<+\infty, p \neq 0$, we define

$$
w_{p}(K)=\left(\int_{S^{n-1}} h_{K}(\theta)^{p} d \sigma(\theta)\right)^{1 / p}
$$


where $h_{K}(\theta)=\max _{x \in K}\langle x, \theta\rangle$ denotes the support function of $K$ in the direction $\theta \in S^{n-1}$. If $|K|=1$, then for all $q \geq 1$, the $L_{q}$-centroid body of $K$ is defined to be the symmetric convex body in $\mathbb{R}^{n}$ whose support function is given by

$$
h_{Z_{q}(K)}(\theta)=\left(\int_{K}|\langle x, \theta\rangle|^{q} d x\right)^{1 / q} .
$$

In [26], Paouris also established the following asymptotic formula for the negative moments $I_{-q}(K, F)$ (see [26, Propositions 5.4 and 4.1]):

Proposition 2.1 (Paouris, [26]). Let $K \subset \mathbb{R}^{n}$ be a convex body with $|K|=1$ and centroid at the origin. Let $k \in\{1, \ldots, n\}$ and $F \in G_{n, k}$. Then, for every integer $q<k$ it holds

$$
I_{-q}(K, F) \simeq \sqrt{\frac{k}{q}} w_{-q}\left(P_{F} Z_{q}(K)\right)
$$

\section{Some PRELIMINARY LEMMAS}

We state here some preliminary technical results which will be needed in the proofs of the main theorems. The first observation states the monotonicity of the family of mean outer radii $\widetilde{R}_{k}$ in $k$.

Lemma 3.1. Let $K \subset \mathbb{R}^{n}$ be a convex body. Then $\widetilde{R}_{k-1}(K) \leq \widetilde{R}_{k}(K), 2 \leq k \leq n$.

Proof. Let $F \in G_{n, k}$, with $k \geq 2$. Then, for any $(k-1)$-dimensional subspace $E$ of $F$, we have that $R\left(P_{E} K\right) \leq R\left(P_{F} K\right)$. Thus

$$
\int_{G_{F, k-1}} R\left(P_{E} K\right) d \nu_{F, k-1}(E) \leq R\left(P_{F} K\right),
$$

and hence

$$
\int_{G_{n, k}} \int_{G_{F, k-1}} R\left(P_{E} K\right) d \nu_{F, k-1}(E) d \nu_{n, k}(F) \leq \int_{G_{n, k}} R\left(P_{F} K\right) d \nu_{n, k}(F)=\widetilde{R}_{k}(K) .
$$

By the uniqueness of the Haar probability measure on $G_{n, k-1}$, the integral on the left hand side of the previous inequality is

$$
\int_{G_{n, k-1}} R\left(P_{E} K\right) d \nu_{n, k-1}(E)=\widetilde{R}_{k-1}(K) .
$$

The following lemmas will be needed to estimate from above the mean outer radii of $K_{N}$ with high probability, for which we will follow the arguments from [10] and [11], used there to estimate the normalized quermassintegrals of $K_{N}$.

Lemma 3.2. Let $K \subset \mathbb{R}^{n}$ be an isotropic convex body and $K_{N}$ be a random polytope generated by $N \geq n$ independent random vectors $X_{1}, \ldots, X_{N}$ uniformly distributed in $K$. Then, for all $1 \leq k \leq n, t>1$ and $q \geq \log N$,

$$
\widetilde{R}_{k}\left(K_{N}\right) \leq c t\left(\int_{G_{n, k}} I_{q}(K, F)^{q} d \nu_{n, k}(F)\right)^{1 / q}
$$

with probability greater than $1-t^{-q}$, where $c>0$ is an absolute constant. 
Proof. Applying Hölder's inequality and Cauchy-Schwartz's inequality we get

$$
\begin{aligned}
\widetilde{R}_{k}\left(K_{N}\right) & =\int_{G_{n, k}} R\left(P_{F} K_{N}\right) d \nu_{n, k}(F) \leq\left(\int_{G_{n, k}} R\left(P_{F} K_{N}\right)^{q / 2} d \nu_{n, k}(F)\right)^{2 / q} \\
& \leq\left(\int_{G_{n, k}} I_{q}(K, F)^{q} d \nu_{n, k}(F)\right)^{1 / q}\left(\int_{G_{n, k}} \frac{R\left(P_{F} K_{N}\right)^{q}}{I_{q}(K, F)^{q}} d \nu_{n, k}(F)\right)^{1 / q} .
\end{aligned}
$$

So, in order to conclude the proof, it suffices to show that

$$
\left(\int_{G_{n, k}} \frac{R\left(P_{F} K_{N}\right)^{q}}{I_{q}(K, F)^{q}} d \nu_{n, k}(F)\right)^{1 / q} \leq c t
$$

with probability greater than $1-t^{-q}$, where $c>0$ is an absolute constant.

In order to prove (5) we notice that, on the one hand, since $K$ is isotropic then for every $F \in G_{n, k}$ it holds $R(K) \leq(n+1) L_{K}$ (see, for instance, [19]), and together with the definition of isotropic constant and Hölder's inequality we get

$$
R\left(P_{F} K_{N}\right) \leq R(K) \leq(n+1) L_{K} \leq \frac{n+1}{\sqrt{k}} I_{q}(K, F) .
$$

Therefore,

$$
\begin{aligned}
\mathbb{E} \int_{G_{n, k}} \frac{R\left(P_{F} K_{N}\right)^{q}}{I_{q}(K, F)^{q}} d \nu_{n, k}(F) & \\
= & \mathbb{E} \int_{0}^{+\infty} q t^{q-1} \nu_{n, k}\left\{F \in G_{n, k}: R\left(P_{F} K_{N}\right) \geq t I_{q}(K, F)\right\} d t \\
& =\int_{0}^{\frac{n+1}{\sqrt{k}}} q t^{q-1} \mathbb{E} \nu_{n, k}\left\{F \in G_{n, k}: R\left(P_{F} K_{N}\right) \geq t I_{q}(K, F)\right\} d t .
\end{aligned}
$$

On the other hand, since $R\left(P_{F} K_{N}\right)=\max _{1 \leq j \leq N}\left|P_{F} X_{j}\right|$ for every $F \in G_{n, k}$, using Markov's inequality we get that for any $1 \leq j \leq N$,

$$
\mathbb{P}\left(\left|P_{F} X_{j}\right| \geq t I_{q}(K, F)\right) \leq t^{-q}
$$

and thus the union bound gives

$$
\mathbb{P}\left(R\left(P_{F} K_{N}\right) \geq t I_{q}(K, F)\right) \leq N t^{-q} .
$$

By a standard application of Fubini's theorem we get that

$$
\begin{aligned}
\mathbb{E} \nu_{n, k}\left\{F \in G_{n, k}:\right. & \left.R\left(P_{F} K_{N}\right) \geq t I_{q}(K, F)\right\} \\
& =\int_{G_{n, k}} \mathbb{P}\left(R\left(P_{F} K_{N}\right) \geq t I_{q}(K, F)\right) d \nu_{n, k}(F) \leq N t^{-q} .
\end{aligned}
$$

This, together with (6) show that for any $A \in(0,(n+1) / \sqrt{k})$ it holds

$$
\begin{aligned}
\mathbb{E} \int_{G_{n, k}} \frac{R\left(P_{F} K_{N}\right)^{q}}{I_{q}(K, F)^{q}} d \nu_{n, k}(F) & \leq \int_{0}^{A} q t^{q-1} d t+\int_{A}^{\frac{n+1}{\sqrt{k}}} q t^{q-1} N t^{-q} d t \\
& =A^{q}+q N \log \left(\frac{n+1}{A \sqrt{k}}\right) .
\end{aligned}
$$

Taking $A=e$ and $q \geq \log N$ we obtain that this is bounded from above by $c^{q}$ with $c$ an absolute constant. Inequality (5) now follows from Markov's inequality. It completes the proof of the lemma. 
Lemma 3.3. Let $K \subset \mathbb{R}^{n}$ be a convex body, $1 \leq k \leq n$ and $q \geq 1$. Then

$$
\left(\int_{G_{n, k}} I_{q}(K, F)^{q} d \nu_{n, k}(F)\right)^{1 / q} \simeq \sqrt{\frac{k+q}{n+q}} I_{q}(K) .
$$

Proof. In [24] it was shown that for any $x \in \mathbb{R}^{n}$ and all $q \geq 1$ it holds

$$
|x| \simeq \sqrt{\frac{n+q}{q}}\left(\int_{S^{n-1}}|\langle x, \theta\rangle|^{q} d \sigma(\theta)\right)^{1 / q} .
$$

Applying Fubini's theorem, the above formula and the uniqueness of the Haar measure on $S^{n-1}$ we obtain

$$
\begin{aligned}
&\left(\int_{G_{n, k}} I_{q}(K, F)^{q} d \nu_{n, k}(F)\right)^{1 / q}=\left(\int_{K} \int_{G_{n, k}}\left|P_{F} x\right|^{q} d \nu_{n, k}(F) d x\right)^{1 / q} \\
& \\
& \simeq \sqrt{\frac{k+q}{q}}\left(\int_{K} \int_{G_{n, k}} \int_{S_{F}^{k-1}}\left|\left\langle P_{F} x, \theta\right\rangle\right|^{q} d \sigma_{F}(\theta) d \nu_{n, k}(F) d x\right)^{1 / q} \\
&=\sqrt{\frac{k+q}{q}}\left(\int_{K} \int_{G_{n, k}} \int_{S_{F}^{k-1}}|\langle x, \theta\rangle|^{q} d \sigma_{F}(\theta) d \nu_{n, k}(F) d x\right)^{1 / q} \\
&=\sqrt{\frac{k+q}{q}}\left(\int_{K} \int_{S^{n-1}}|\langle x, \theta\rangle|^{q} d \sigma_{n-1}(\theta) d x\right)^{1 / q} \\
& \simeq \sqrt{\frac{k+q}{n+q}}\left(\int_{K}|x|^{q} d x\right)^{1 / q}=\sqrt{\frac{k+q}{n+q}} I_{q}(K) .
\end{aligned}
$$

The following lemmas will be used for estimating the lower bound in Theorem 1.1. The method will be similar to the one for the upper bound, but involving the negative moments of the Euclidean norm.

Lemma 3.4. Let $K \subset \mathbb{R}^{n}$ be a convex body and $K_{N}$ be a random polytope generated by $N \geq n$ independent random vectors $X_{1}, \ldots, X_{N}$ uniformly distributed in $K$. Then, for any $1 \leq k \leq n, q<k$ and $t \in(0,1)$,

$$
\left(\int_{G_{n, k}} \frac{R\left(P_{F} K_{N}\right)^{-q}}{I_{-q}(K, F)^{-q}} d \nu_{n, k}(F)\right)^{-1 / q} \geq t\left(\frac{N-1}{N}\right)^{1 / q}
$$

with probability greater than $1-t^{q}$.

Proof. Since $R\left(P_{F} K_{N}\right)=\max _{1 \leq j \leq N}\left|P_{F} X_{j}\right|$ for every $F \in G_{n, k}$, using Markov's inequality we get that for any $1 \leq j \leq N$ and all $q<k$,

$$
\mathbb{P}\left(\left|P_{F} X_{j}\right| \leq \varepsilon I_{-q}(K, F)\right)=\mathbb{P}\left(\left|P_{F} X_{j}\right|^{-q} \geq \varepsilon^{-q} I_{-q}(K, F)^{-q}\right) \leq \varepsilon^{q},
$$

for every $\varepsilon \in(0,1)$, and thus

$$
\mathbb{P}\left(R\left(P_{F} K_{N}\right) \leq \varepsilon I_{-q}(K, F)\right) \leq \varepsilon^{N q} .
$$

Then, a standard application of Fubini's theorem leads to

$$
\mathbb{E} \nu_{n, k}\left\{F \in G_{n, k}: R\left(P_{F} K_{N}\right) \leq \varepsilon I_{-q}(K, F)\right\} \leq \varepsilon^{N q}, \quad \varepsilon \in(0,1),
$$


which can be used to bound the expectation from above:

$$
\begin{aligned}
\mathbb{E} \int_{G_{n, k}} \frac{R\left(P_{F} K_{N}\right)^{-q}}{I_{-q}(K, F)^{-q}} d \nu_{n, k}(F) & \\
= & \mathbb{E} \int_{0}^{+\infty} q t^{q-1} \nu_{n, k}\left\{F \in G_{n, k}: I_{-q}(K, F) \geq t R\left(P_{F} K_{N}\right)\right\} d t \\
& =\int_{0}^{+\infty} q t^{q-1} \mathbb{E} \nu_{n, k}\left\{F \in G_{n, k}: R\left(P_{F} K_{N}\right) \leq \frac{1}{t} I_{-q}(K, F)\right\} d t \\
& \leq \int_{0}^{1} q t^{q-1} d t+\int_{1}^{+\infty} q t^{q-1-N q} d t=1+\frac{1}{N-1}=\frac{N}{N-1} .
\end{aligned}
$$

To finish the proof we apply Markov's inequality.

In the next lemma, Proposition 2.1 plays a crucial role.

Lemma 3.5. Let $K \subset \mathbb{R}^{n}$ be a convex body with $|K|=1$ and centroid at the origin. For every $1 \leq k \leq n$ and every $q<k$ it holds

$$
\left(\int_{G_{n, k}} I_{-q}(K, F)^{-q} d \nu_{n, k}(F)\right)^{-1 / q} \simeq \sqrt{\frac{k}{n}} I_{-q}(K) .
$$

Proof. Using Proposition 2.1 and the uniqueness of the Haar probability measure on the sphere $S^{n-1}$, we get

$$
\begin{aligned}
\left(\int_{G_{n, k}} I_{-q}(K, F)^{-q}\right. & \left.d \nu_{n, k}(F)\right)^{-1 / q} \simeq \sqrt{\frac{k}{q}}\left(\int_{G_{n, k}} w_{-q}\left(P_{F} Z_{q}(K)\right)^{-q} d \nu_{n, k}(F)\right)^{-1 / q} \\
& =\sqrt{\frac{k}{q}}\left(\int_{G_{n, k}} \int_{S_{F}^{k-1}} h_{P_{F} Z_{q}(K)}(\theta)^{-q} d \sigma_{F}(\theta) d \nu_{n, k}(F)\right)^{-1 / q} \\
& =\sqrt{\frac{k}{q}}\left(\int_{G_{n, k}} \int_{S_{F}^{k-1}} h_{Z_{q}(K)}(\theta)^{-q} d \sigma_{F}(\theta) d \nu_{n, k}(F)\right)^{-1 / q} \\
& =\sqrt{\frac{k}{q}}\left(\int_{S^{n-1}} h_{Z_{q}(K)}(\theta)^{-q} d \sigma(\theta)\right)^{-1 / q}=\sqrt{\frac{k}{q}} w_{-q}\left(Z_{q}(K)\right) .
\end{aligned}
$$

Applying again Proposition 2.1 we get the result.

\section{Asymptotic Formula for the Mean Outer Radil of Random POLYTOPES}

Now we are ready to prove Theorem 1.1.

Proof of Theorem 1.1. First we state the upper bound for the mean outer radii of $K_{N}$ in the full range $n \leq N \leq e^{\sqrt{n}}$. Applying Lemma 3.2 and Lemma 3.3 with $q=\log N$ and $t=e^{s}$ for any $s>0$, we get that

$$
\widetilde{R}_{k}\left(K_{N}\right) \leq c(s) \sqrt{\frac{k+\log N}{n+\log N}} I_{\log N}(K)
$$


with probability greater than $1-N^{-s}$, where $c(s)>0$ is an absolute constant depending only on $s$. Using (3) for $q=\log N$, and since $N \leq e^{\sqrt{n}}$, we obtain that $I_{\log N}(K) \leq c \sqrt{n} L_{K}$. Consequently, we get

$$
\widetilde{R}_{k}\left(K_{N}\right) \leq c(s) \sqrt{k+\log N} L_{K} \simeq \max \{\sqrt{k}, \sqrt{\log N}\} L_{K} .
$$

Next we show the lower bound in Theorem 1.1. We start assuming that $\log N<$ $k, 1 \leq k \leq n$. By Hölder's inequality,

$$
\begin{aligned}
\widetilde{R}_{k}\left(K_{N}\right) & =\int_{G_{n, k}} R\left(P_{F} K_{N}\right) d \nu_{n, k}(F) \geq\left(\int_{G_{n, k}} R\left(P_{F} K_{N}\right)^{-q / 2} d \nu_{n, k}(F)\right)^{-2 / q} \\
& \geq\left(\int_{G_{n, k}} I_{-q}(K, F)^{-q} d \nu_{n, k}(F)\right)^{-1 / q}\left(\int_{G_{n, k}} \frac{R\left(P_{F} K_{N}\right)^{-q}}{I_{-q}(K, F)^{-q}} d \nu_{n, k}(F)\right)^{-1 / q},
\end{aligned}
$$

and then Lemmas 3.4 and 3.5 for $q=\log N<k$ and $t=e^{-s}$ imply that

$$
\widetilde{R}_{k}\left(K_{N}\right) \geq c(s) \sqrt{\frac{k}{n}} I_{-\log N}(K)
$$

with probability greater than $1-N^{-s}$, where $c(s)$ is an absolute constant depending only on $s$. Since $\log N \leq \sqrt{n}$, using (4) we get that $I_{-\log N}(K) \simeq \sqrt{n} L_{K}$ and the result follows.

In [11] it was shown that $\left(\left|K_{N}\right| /\left|B_{2}^{n}\right|\right)^{1 / n} \geq c(s) \sqrt{\log (N / n)} L_{K}$ with probability greater than $1-N^{-s}$. The monotonicity of the mean outer radii (Lemma 3.1) and the fact that $\left(\left|K_{N}\right| /\left|B_{2}^{n}\right|\right)^{1 / n} \leq \widetilde{R}_{1}\left(K_{N}\right)$ complete the proof of this case.

Remark 4.1. In order to obtain the upper bound for the mean outer radii in Theorem 1.1 we cannot expect to extend the range of $N$ to $N=e^{\beta n}$ for some $\beta \in(0,1)$. In fact, if this holds for, say, $\beta=1 / 2$, then in the case $k=1$ we get that for every isotropic convex body $K \subset \mathbb{R}^{n}$, we have

$$
\mathbb{P}\left(\omega\left(K_{N}\right) \leq \widetilde{R}_{1}\left(K_{N}\right) \leq \sqrt{n} L_{K}\right) \geq 1-e^{-n / 2} .
$$

Since it is known (see [13]) that for $N \simeq e^{n / 2}$ it holds

$$
\mathbb{P}\left(K_{N} \subseteq K \subseteq c(\delta) K_{N}\right) \geq 1-\delta,
$$

then choosing the parameters $(\delta>1 / 2, n \geq 2)$ we get that the event

$$
\left\{\omega\left(K_{N}\right) \leq \sqrt{n} L_{K} \text { and } K \subseteq c K_{N}\right\}
$$

occurs with positive probability. Thus, for every isotropic convex body $K \subset \mathbb{R}^{n}$, $\omega(K) \leq c \omega\left(K_{N}\right) \leq C \sqrt{n} L_{K}$, which is not true, as the cross-polytope shows.

Regarding an extension of the range of $N$ from below, we note that similar arguments work also for $n^{1+\delta}<N<e^{\sqrt{n}}$, for any $\delta>0$, just replacing the constants $c_{1}(\delta)$ and $c_{2}(\delta)$. However, if the number of vertices $N$ is proportional to the dimension $n$, i.e., $N \simeq n$, more refined arguments are needed.

Now we show that if the expectation is involved, the range for $N$ can be extended from below till $N \geq n$. In the proof we will need the following result which was shown in [3]: in our notation, if $K \subset \mathbb{R}^{n}$ is an isotropic convex body and $X_{1}, \ldots, X_{N}$ are independent random vectors uniformly distributed in $K$, then for every $n \leq N \leq$ $e^{\sqrt{n}}$ we have

$$
\mathbb{E} \widetilde{R}_{1}\left(K_{N}\right) \simeq \sqrt{\log N} L_{K} .
$$


Remark 4.2. Since in [3] the authors were interested in studying the value of the mean width of $K_{N}$, which is the same as $\widetilde{R}_{1}\left(K_{N}\right)$ only when $K_{N}$ is symmetric, equation (8) is specifically written only in the symmetric case. However, the same proof leads to this equation in the non-symmetric case.

Proof of Theorem 1.2. First we use the fact that the upper bound in Theorem 1.1 is valid for the whole range $n \leq N \leq e^{\sqrt{n}}$. Thus, for every $1 \leq k \leq n$ and all $s>0$,

$$
\mathbb{E} \widetilde{R}_{k}\left(K_{N}\right) \leq c(s) \max \{\sqrt{k}, \sqrt{\log N}\} L_{K}+N^{-s} R(K) .
$$

Since $R(K) \leq(n+1) L_{K}$ and taking $s=1$, we obtain, on the one hand, that

$$
\mathbb{E} \widetilde{R}_{k}\left(K_{N}\right) \leq c \max \{\sqrt{k}, \sqrt{\log N}\} L_{K} .
$$

On the other hand, by Markov's inequality, we have that for every $\alpha>0$

$$
\mathbb{E} \widetilde{R}_{k}\left(K_{N}\right) \geq \alpha \mathbb{P}\left(\widetilde{R}_{k}\left(K_{N}\right) \geq \alpha\right) .
$$

From equation (7) we have

$$
\mathbb{E} \widetilde{R}_{k}\left(K_{N}\right) \geq c(s) \sqrt{k} L_{K}\left(1-N^{-s}\right) \geq c \sqrt{L}_{K}
$$

choosing $s$ to be some absolute constant. Finally, using (8) and the monotonicity of the mean outer radii (Lemma 3.1), we have that, for all $1 \leq k \leq n$ and $n<N<$ $e^{\sqrt{n}}$

$$
\mathbb{E} \widetilde{R}_{k}\left(K_{N}\right) \geq \mathbb{E} \widetilde{R}_{1}\left(K_{N}\right) \simeq \sqrt{\log N} L_{K} .
$$

The theorem follows from (9), (10) and (11).

\section{The Gaussian Case}

In this last section we consider the case of Gaussian random polytopes, and show Theorem 1.3. We observe that, since the distribution of a Gaussian vector is rotationally invariant and its projection on any $k$-dimensional subspace is a $k$ dimensional Gaussian vector, it is a direct consequence of the following result for the orthogonal projection of Gaussian random vectors:

Proposition 5.1. Let $G_{1}, \ldots, G_{N}$ be independent standard Gaussian random vectors in $\mathbb{R}^{k}$. Then

$$
\mathbb{E} \max _{1 \leq j \leq N}\left|G_{j}\right| \simeq \max \{\sqrt{k}, \sqrt{\log N}\} .
$$

Although this result is probably known by specialists, we include a proof, since we were not able to find one in the literature except for the 1-dimensional case (see [22]). First we need the following lemma:

Lemma 5.1. Let $k \geq 1$ and $t \geq \max \{\sqrt{2(k-1)}, 1\}$. Then

$$
t^{k-1} e^{-\frac{t^{2}}{2}} \leq \int_{t}^{\infty} r^{k} e^{-\frac{r^{2}}{2}} d r \leq 2 t^{k-1} e^{-\frac{t^{2}}{2}} .
$$

Proof. Since $t \geq 1$

$$
\int_{t}^{\infty} r^{k} e^{-\frac{r^{2}}{2}} d r \geq t^{k-1} \int_{t}^{\infty} r e^{-\frac{r^{2}}{2}} d r=t^{k-1} e^{-\frac{t^{2}}{2}}
$$


which shows the left inequality. In order to prove the right hand side inequality, we consider the function

$$
f(t)=2 t^{k-1} e^{-\frac{t^{2}}{2}}-\int_{t}^{\infty} r^{k} e^{-\frac{r^{2}}{2}} d r
$$

Since $f^{\prime}(t)=t^{k-2} e^{-\frac{t^{2}}{2}}\left(2(k-1)-t^{2}\right), f(t)$ decreases if $t \geq \sqrt{2(k-1)}$ which, together with the fact that $\lim _{t \rightarrow \infty} f(t)=0$, gives the result.

Proof of Proposition 5.1. For $k=1$ the result is well known, so we assume that $k \geq 2$. Integrating in polar coordinates and taking into account that $k \geq 2$, we have

$$
\begin{aligned}
\mathbb{P}\left(\max _{1 \leq j \leq N}\left|G_{j}\right| \leq t\right) & =\mathbb{P}\left(\left|G_{j}\right| \leq t\right)^{N}=\left(k\left|B_{2}^{k}\right| \int_{0}^{t} r^{k-1} \frac{e^{-\frac{r^{2}}{2}}}{(\sqrt{2 \pi})^{k}} d r\right)^{N} \\
& =\left(1-k\left|B_{2}^{k}\right| \int_{t}^{\infty} r^{k-1} \frac{e^{-\frac{r^{2}}{2}}}{(\sqrt{2 \pi})^{k}} d r\right)^{N} \\
& \leq\left(1-k\left|B_{2}^{k}\right| t^{k-2} \int_{t}^{\infty} r \frac{e^{-\frac{r^{2}}{2}}}{(\sqrt{2 \pi})^{k}} d r\right)^{N} \\
& =\left(1-k\left|B_{2}^{k}\right| t^{k-2} \frac{e^{-\frac{t^{2}}{2}}}{(\sqrt{2 \pi})^{k}}\right)^{N \log \left(1-k\left|B_{2}^{k}\right| t^{k-2} \frac{e^{-\frac{t^{2}}{2}}}{(\sqrt{2 \pi})^{k}}\right)} \\
& \leq e^{-N k\left|B_{2}^{k}\right| t^{k-2} \frac{e^{-\frac{t^{2}}{2}}}{(\sqrt{2 \pi})^{k}}}
\end{aligned}
$$

Now, using the well-known value of the volume of the Euclidean unit ball, namely, $\left|B_{2}^{k}\right|=\pi^{k / 2} / \Gamma(1+k / 2)$, by Stirling's formula we have that there exists an absolute constant $c$ such that

$$
\mathbb{P}\left(\max _{1 \leq j \leq N}\left|G_{j}\right| \leq t\right) \leq e^{-c \frac{N \sqrt{k}}{t^{2}}\left(\frac{\sqrt{e} t}{\sqrt{k}}\right)^{k} e^{-\frac{t^{2}}{2}}} .
$$

Thus, taking $t=\sqrt{k}$ we obtain that

$$
\mathbb{P}\left(\max _{1 \leq j \leq N}\left|G_{j}\right| \leq \sqrt{k}\right) \leq e^{-c \frac{N}{\sqrt{k}}} \leq e^{-c \sqrt{n}},
$$

which tends to 0 when $n \rightarrow \infty$. Hence, there exists $n_{0} \in \mathbb{N}$ such that if $n \geq n_{0}$,

$$
\mathbb{P}\left(\max _{1 \leq j \leq N}\left|G_{j}\right|>\sqrt{k}\right) \geq \frac{1}{2}
$$

and so, if $n \geq n_{0}$,

$$
\mathbb{E} \max _{1 \leq j \leq N}\left|G_{j}\right| \geq \sqrt{k} \mathbb{P}\left(\max _{1 \leq j \leq N}\left|G_{j}\right| \geq \sqrt{k}\right)>\frac{\sqrt{k}}{2} .
$$

This shows the lower estimate for the expectation when $k \geq e \log N$. Otherwise, taking $t=\sqrt{\log N}$ we obtain

$$
\mathbb{P}\left(\max _{1 \leq j \leq N}\left|G_{j}\right| \leq \sqrt{\log N}\right) \leq e^{-c \frac{\sqrt{N} \sqrt{k}}{\log N}\left(\frac{\sqrt{e \log N}}{\sqrt{k}}\right)^{k}} \leq e^{-c \frac{\sqrt{N}}{\log N}}
$$


because $1 \leq k \leq e \log N$. Thus, there exists $n_{0} \in \mathbb{N}$ such that if $n \geq n_{0}$,

$$
\mathbb{P}\left(\max _{1 \leq j \leq N}\left|G_{j}\right| \geq \sqrt{\log N}\right) \geq \frac{1}{2}
$$

and therefore, if $n \geq n_{0}$ and $1 \leq k \leq e \log N$,

$$
\mathbb{E} \max _{1 \leq j \leq N}\left|G_{j}\right| \geq \sqrt{\log N} \mathbb{P}\left(\max _{1 \leq j \leq N}\left|G_{j}\right| \geq \sqrt{\log N}\right) \geq \frac{\sqrt{\log N}}{2} .
$$

On the other hand, for any $A>0$,

$$
\begin{aligned}
\mathbb{E} \max _{1 \leq j \leq N}\left|G_{j}\right| & =\int_{0}^{\infty} \mathbb{P}\left(\max _{1 \leq j \leq N}\left|G_{j}\right| \geq t\right) d t \\
& =\int_{0}^{A} \mathbb{P}\left(\max _{1 \leq j \leq N}\left|G_{j}\right| \geq t\right) d t+\int_{A}^{\infty} \mathbb{P}\left(\max _{1 \leq j \leq N}\left|G_{j}\right| \geq t\right) d t \\
& \leq A+N \int_{A}^{\infty} \mathbb{P}\left(\left|G_{1}\right| \geq t\right) d t \\
& =A+N k\left|B_{2}^{k}\right| \int_{A}^{\infty} \int_{t}^{\infty} r^{k-1} \frac{e^{-\frac{r^{2}}{2}}}{(\sqrt{2 \pi})^{k}} d r d t .
\end{aligned}
$$

Taking $A=\max \{2 \sqrt{\log N}, \sqrt{2 k}\}$ we get that

$$
\begin{aligned}
\mathbb{E} \max _{1 \leq j \leq N}\left|G_{j}\right| & \leq A+2 N k\left|B_{2}^{k}\right| \int_{A}^{\infty} t^{k-2} \frac{e^{-\frac{t^{2}}{2}}}{(\sqrt{2 \pi})^{k}} d t \\
& \leq A+4 N k\left|B_{2}^{k}\right| A^{k-3} \frac{e^{-\frac{A^{2}}{2}}}{(\sqrt{2 \pi})^{k}} \leq A+\frac{C N \sqrt{k}}{A^{3}}\left(\frac{\sqrt{e} A}{\sqrt{k}}\right)^{k} e^{-\frac{A^{2}}{2}} .
\end{aligned}
$$

If $A=2 \sqrt{\log N}$ it can be checked that

$$
\mathbb{E} \max _{1 \leq j \leq N}\left|G_{j}\right| \leq 2 \sqrt{\log N}+\frac{C}{N \log N} 2 \sqrt{e} \sqrt{\log N} \leq C \sqrt{\log N} .
$$

Finally, if $A=\sqrt{2 k}$, then

$$
\mathbb{E} \max _{1 \leq j \leq N}\left|G_{j}\right| \leq \sqrt{2 k}+\frac{C N}{k} e^{-\frac{k}{2}} \leq \sqrt{2 k}+\frac{C}{k} \leq C \sqrt{k} .
$$

\section{REFERENCES}

[1] D. Alonso-Gutiérrez, On the isotropy constant of random convex sets, Proc. Amer. Math. Soc. 136 (9) (2008), 3293-3300.

[2] D. Alonso-Gutiérrez, J. Prochno, Estimating Support Functions of Random Polytopes via Orlicz Norms, Discrete Comput. Geom., DOI 10.1007/s00454-012-9468-7, 2012.

[3] D. Alonso-Gutiérrez, J. Prochno, On the Gaussian Behavior of Marginals and the Mean Width of Random Polytopes, preprint (2012).

[4] I. BÁRÁNY, Random polytopes, convex bodies, and approximation, Stochastic Geometry, Lecture Notes in Math. 1892 (2007), 77-118.

[5] U. Betke, M. Henk, Estimating sizes of a convex body by successive diameters and widths, Mathematika 39 (2) (1992), 247-257.

[6] K.-H. Borgwardt, The simplex method. A probabilistic analysis, Algorithms and Combinatorics: Study and Research Texts 1, Springer-Verlag, Berlin, 1987.

[7] R. Brandenberg, Radii of regular polytopes, Discrete Comput. Geom. 33 (1) (2005), 43-55.

[8] R. Brandenberg, T. Theobald, Radii minimal projections of polytopes and constrained optimization of symmetric polynomials, Adv. Geom. 6 (1) (2006), 71-83. 
[9] N. Dafnis, A. Giannopoulos, O. Guédon, On the isotropic constant of random polytopes, Adv. Geom. 10 (2010), 311-32.

[10] N. Dafnis, A. Giannopoulos, A. Tsolomitis, Asymptotic shape of a random polytope in a convex body, J. Funct. Anal. 257 (2009), 2820-2839.

[11] N. Dafnis, A. Giannopoulos, A. Tsolomitis, Quermaßintegrals and asymptotic shape of random polytopes in an isotropic convex body, Michigan Math. J. (to appear).

[12] A. Giannopoulos, Notes on isotropic convex bodies, Warsaw University Notes, 2003.

[13] A. Giannopoulos, V. Milman, Concentration property on probability spaces, Adv. Math. 156 (2000), 77-106.

[14] B. González, M. A. Hernández Cifre, Successive radii and Minkowski addition, Monatsh. Math. 166 (2012), 395-409.

[15] B. González, M. A. Hernández Cifre, A. Hinrichs, Successive radii of families of convex bodies, Submitted (2012).

[16] P. Gritzmann, V. Klee, Computational complexity of inner and outer $j$-radii of polytopes in finite-dimensional normed spaces, Math. Program. 59 (1993) 163-213.

[17] M. Henk, M. A. Hernández Cifre, Intrinsic volumes and successive radii, J. Math. Anal. Appl. 343 (2) (2008), 733-742.

[18] M. Henk, M. A. Hernández Cifre, Successive minima and radii, Canad. Math. Bull. 52 (3) (2009), 380-387.

[19] R. Kannan, L. Lovsz, M. Simonovits, Isoperimetric problems for convex bodies and a localization lemma, Discrete Comp. Geom. 3-4 (1995), 541-559.

[20] B. Klartag, G. Kozma, On the hyperplane conjecture for random convex sets, Israel J. Math. 170 (2009), 253-268.

[21] B. Klartag, E. Milman, Centroid bodies and the logarithmic Laplace transform - a unified approach, J. Funct. Anal. 262 (1) (2012), 10-34.

[22] M. Ledoux, M. Talagrand, Probability in Banach spaces. Isoperimetry and processes, Reprint of the 1991 edition. Classics in Mathematics, Springer-Verlag, Berlin, 2011.

[23] V. D. Milman, A. PAJor, Isotropic position and inertia ellipsoids and zonoids of the unit ball of a normed $n$-dimensional space, Geometric aspects of Functional Analysis, Lecture Notes in Math. 1376 (1989), 64-104.

[24] G. PAOuRIS, $\psi_{2}$-estimates for linear functionals on zonoids, Geometric aspects of Functional Analysis, Lecture Notes in Math. 1807 (2003), 211-222.

[25] G. Paouris, Concentration of mass on convex bodies, Geom. Funct. Anal. 16 (5) (2006), 1021-1049.

[26] G. Paouris, Small ball probability estimates for log-concave measures, Trans. Amer. Math. Soc. 364 (2012), 287-308.

[27] G. Paouris, P. Pivovarov, A probabilistic take on isoperimetric-type inequalities, Adv. Math. 230 (3) (2012), 1402-1422.

[28] F. P. Preparata, M. I. Shamos, Computational geometry. An introduction, Texts and Monographs in Computer Science, Springer-Verlag, New York, 1985.

[29] S. V. Puhov, Inequalities for the Kolmogorov and Bernštern widths in Hilbert space, Math. Notes 25 (4) (1979), 320-326.

[30] M. Reitzner, Random polytopes, New perspectives in stochastic geometry, Oxford University Press, Oxford, 2010, 45-76.

[31] A. RÉnyi, R. Sulanke, Über die konvexe Hülle von $n$ zufällig gewählten Punkten, $Z$. Wahrsch. Verw. Geb. 2 (1963), 75-84.

[32] H. Solomon, Geometric probability, CBMSRegional Conference Series in Applied Mathematics 28, Society for Industrial and Applied Mathematics, Philadelphia, Pa., 1978. 
Departamento de Matemáticas, Universidad de Murcia, Campus de Espinardo, 30100Murcia, Spain

E-mail address: davidalonso@um.es; mhcifre@um.es

Department of Mathematics, Texas A\&M University, College Station, TX 77843 U.S.A.

E-mail address: nikdafnis@gmail.com

Institute of Analysis, Johannes Kepler University Linz, Altenbergerstr. 69, A-4040 Linz, Austria

E-mail address: joscha.prochno@jku.at 